\title{
Strain Quantification In Ultrasound Sequences
}

\author{
J.D. Revell ${ }^{1}$, M. Mirmehdi ${ }^{1}$ and D.S. McNally ${ }^{2}$ \\ ${ }^{1}$ Department of Computer Science, University of Bristol, Bristol, BS8 1UB, UK \\ ${ }^{2}$ Institute of Biomechanics, University of Nottingham, Nottingham, NG7 2RD, UK \\ \{revell, majid\}@cs.bris.ac.uk, Donal.Mcnally@nottingham.ac.uk
}

\begin{abstract}
A novel methodology to quantify displacements and strain in musculoskeletal ultrasound sequences is presented. We extend the principles of 2D interframe displacements produced by our earlier work using hierarchical variable block size matching, to quantify displacement trajectories. We provide novel solutions for probe motion, quantification of objects moving in the 3D volume traversing the $2 \mathrm{D}$ plane, and improving the temporal coherence of displacements for longer image sequences than the frame pairs traditionally applied in ultrasound. We also present trajectory strain that yields a novel strain history for musculoskeletal tendon tissue samples.
\end{abstract}

\section{Introduction}

Observing the dynamic responses of soft tissues permits the inference of physical properties of tissues, such as elasticity and strain, as well as aiding in diagnosis. This is the principle of sonoelastography, which derives the biomechanical properties of tissue from measurements of motion resulting from an applied perturbation, by estimating local axial strain using cross correlation analysis on RF A-lines [10]. This approach is very dependent upon the hardware of the imager and requires direct access to the RF signal. Hence current research has favoured towards intrinsic (image-based) methods over extrinsic (invasive) techniques to quantify displacements; more specifically traditional ultrasound motion analysis research has concentrated on analysing specific frame pairs using a variety of methods including optical flow [4], spectral integrals [2] and block differentials [5][1].

We present a novel extension to interframe displacements by quantifying motion trajectories in longer ultrasound sequences (typically 30 frames - limit imposed by acquisition device). Our contributions include, encapsulating increased temporal displacement correlation, probe motion registration and quantifying objects in the $3 \mathrm{D}$ volume traversing in and out of the 2D plane. This is achieved by extending a multiresolution block matching algorithm defined in [7] to compute a trajectory field of tendon tissue matter using normalised cross correlations in the Fourier domain. Trajectory benefits include extending interframe displacements to sequences to produce temporal displacements and consequently quantify gradual incremental and total 3D spatiotemporal strain. Although it has been stated that displacement knowledge yields axial strain maps we have found little research illustrating such results [11]. We illustrate novel temporal strain results from motion trajectories that quantify the strain for a given sample through a sequence. We are not aware of any existing research that attempts to quantify a strain history for regions of soft tissue. These potentially enable clinicians to uniquely visualise and analytically compare soft tissue, incremental strain history and applied loading simultaneously in a 
non invasive system. Throughout, we examine the performance of our proposed speckle tracking system on in vitro and in vivo B-scan data.

During any clinical freehand ultrasound sequence acquisition, both probe and subject are kept stationary to ensure a reproducible imaging plane. Sonographer fatigue, probe decoupling, subject and feature movement can produce observable effects in imaging. Image registration prior to displacement quantification is necessary for invariance to image acquisition-specific artifacts, including fluctuating probe motion (including pressure) that occur in freehand scanning. Variations of probe pressure on the skin can cause local deformations of the anatomy on a large scale compared to pixel size. Global displacements can be derived locally [12] and globally [8] using both intrinsic and extrinsic measures. Without extrinsic probe position measurements and global displacement registration limitations, we have used local measurements in the skin-to-transducer surface region to register our displacement trajectory fields since from our previous work [7] we observed that this region takes on probe motion characteristics.

After briefly outlining our in vitro groundtruth and in vivo datasets in Section 2, we detail our proposed method in Section 3, explaining the logical extension of trajectories from interframe displacements, tracking, trajectory updating, probe motion and aperture correction and temporal strain history. Results from in vitro groundtruth and in vivo musculoskeletal sequences of the patella and achilles tendons are illustrated in Sections 4 and 5. Finally, we discuss the important benefits of trajectories, use of the normalised correlation coefficients as a confidence measure, strain volumetric data and future work.

\section{Datasets: in vitro Groundtruth and in vivo Sequences}

Synthetic images have been commonly used in the past for testing, e.g. [9]. However, we reduce in vivo result ambiguity [2] by using real in vitro groundtruth data that also facilitate better performance evaluation of the proposed method. The first in vitro dataset is an equine tendon with inserted landmarks pulled under controlled loads whilst continuously scanning. The second is a section of cut muscle, and again pulled at various controlled rates. All sequences consist of 30 frames, the maximum acquisition length from existing ultrasound machines, with dynamic capture functionality increasingly being demanded.

Longitudinal sections of normal tendons (in vivo data) were captured with an 8-15 $\mathrm{MHz}$ probe using a Diasus Dynamic Imaging machine. Each sequence captures a dynamic flexion to extension of the muscle giving rise to tendon displacement. The tendon's anisotropic fibrillar texture [3] means that any slight obliquity of the angle of incidence or curvilinearity, can obscure or mimic texture details and create aperture problems. Here we present results on the patella and achilles tendons, areas of high clinical interest due to the demand for improved understanding of tendinopathy typically from athletic injuries. More dynamic (including synthetic groundtruth) results can be found online ${ }^{1}$.

\section{Proposed Method}

In [7] we defined a multiscale block matching pyramid initialised by a regular lattice $R$, sampled by $P \times Q$ (typically $8 \times 8$ or $4 \times 4$ ) on an initial ultrasound frame $f_{t}$. Blocks of

\footnotetext{
${ }^{1}$ http://www.cs.bris.ac.uk/home/revell/flow.html
} 
$M \times N$, where $M, N=\{64,32,16,8,4\}$ were used to increasingly improve displacement accuracy for $R$ until $M=P$ and $N=Q$. Using spatial convolution we minimised the mean-squared error (MSE) between candidate blocks $I$ in a search region $I^{\prime}$ from $f_{t}$ and $f_{t+1}$ respectively, to find the optimum displacement.

\subsection{Motion Trajectories}

Here, instead of the MSE, we determine the local disparity between $I$ and $I^{\prime}$ by identifying the maximum correlation coefficient $c_{\max }$ using normalised cross correlation (NCC). $I$ and $I^{\prime}$ are defined as candidate and reference blocks respectively where $I, I^{\prime}=M \times N$, enabling the NCC to be performed in the frequency domain using the FFT for efficiency:

$$
c=\frac{F^{-1}\left\{\hat{I}^{*} \hat{I}^{\prime}\right\}}{\sqrt{\iint|\hat{I}|^{2} \cdot \iint\left|\hat{I}^{\prime}\right|^{2}}} \quad \text { leading to } \quad\{0 \leq c \leq 1\}
$$

where $\hat{I}$ and $\hat{I}^{\prime}$ denote the Fourier transform of blocks $I$ and $I^{\prime}$ respectively, $F^{-1}$ the inverse Fourier transform and ${ }^{*}$ the complex conjugate. By multiplying the spectral components, the DC element is filtered out removing any global B-mode illumination and high frequency noise. Furthermore, by normalising the correlation, invariance to mean intensity fluctuations is achieved. The spatial displacement vector $\mathbf{d}=\left(d_{x}, d_{y}\right)$ is then estimated in the $x$ and $y$ directions after locating $c_{\max }$. For each block in $R$ the NCC is performed at multiple block scales, using the previous $\mathbf{d}$ as $I$ and $I^{\prime}$ offsets, allowing a varying smoothness constraint across each $I$ for all scales.

Trajectories represent the temporal tracking of features sequentially through a sequence using the NCC for feature location. NCC tracking is sensitive to imaging scale, rotation and perspective distortions. In this context minimal perspective and rotation distortions potentially exist, however, the NCC does enable equal sized patterns to be detected by a rotation distortion of $5^{\circ}$ to $10^{\circ}$ [6]. Image scale distortions are more prominent, and by using the multiscale NCC we achieve scale invariance and improved accuracy from local reflectance variations.

At this stage we have quantified an optimal displacement $\mathbf{d}$ for each block in $f_{t}$ lattice $R$ yielding an interframe displacement field $\mathbf{d}_{R}$. For sequences, this process is repeated for every frame pair in the sequence to give:

$$
\mathbf{d}_{R}^{t} \ldots \mathbf{d}_{R}^{t+n}=\operatorname{NCC}\left\{\left(f_{t}, f_{t+1}\right) \ldots\left(f_{t+n}, f_{t+n+1}\right)\right\}
$$

After quantifying interframe displacements using $R$, we redefine lattice $R$ for the next iteration, deformed by the prior displacement field $\mathbf{d}_{R}^{t}$. Consequent tracking results in a displacement vector with temporal history $h$, a trajectory defined for each original block in $R$ over $h$ steps, hence producing a trajectory field. A powerful benefit from the trajectory definition is $h$ which has a direct relationship with temporal displacement coherence.

Tracking, especially in long sequences, requires feature identification in each next frame $f_{t+1}$ for new objects entering and old objects exiting trajectory updating. Potential causes of such trajectory updates are features traversing in the $2 \mathrm{D}$ plane, 3D volume, at image boundaries and occlusion, producing potential trajectory clusters and voids. A new trajectory is included by comparing each $\mathbf{d}$ to neighbours in $R$ centred in the range $A \times B$, with an Euclidean distance $>P \times Q$. Similarly, trajectories are flagged for removal 
if any neighbouring final positions in $A \times B$ conflict, by a proximity threshold defined empirically. Trajectories in Figs. 2, 3 and 4 are illustrated in black for $R$ and red for updates (for colour images please see online ${ }^{1}$ ).

As inferred in (1) correlation coefficients scale between 0 and 1 denoting a range of weak to strong matches. To measure confidence strength of trajectory displacements we derived a mean confidence measure from the final scale peak correlation coefficients for all blocks in $R$ for frame pairs. These results are discussed and illustrated later in Fig. 7.

\subsection{Displacement Correction}

To correct for the error induced by probe motion $\delta_{x y}$ we used local measurements in the skin-to-transducer surface region (e.g. top most regions in Figs. 3(a)-3(c) and 4(a)-4(c)), by assuming this region corresponded to signal only from the probe. This has been found to be consistent with all data. Hence global displacement and trajectory field registration is achieved by applying the mean displacement, $\delta_{x y}^{\mu}$, in this region, and updating the fields respectively resulting in:

$$
\mathbf{d}=\left(d_{x}+\delta_{x}^{\mu}, d_{y}+\delta_{y}^{\mu}\right)
$$

Given the highly ordered structure of superimposed planes of callogen and septa, tendons appear strongly anisotropic with fibrillar echoes only captured effectively when the probe is perpendicular to them; however, this leads to large aperture problems from tendon movement. To reduce the aperture problem we calculate the anisotropy ratio to locate the initial peak correlation location in the peak region. This is achieved by adaptive thresholding the correlation field $\mathbf{c}_{\text {in }}$ produced from the NCC, to yield $\mathbf{c}_{\text {out }}$, a binary field:

$$
\mathbf{c}_{\text {out }}(i, j)= \begin{cases}0 & \text { if } \mathbf{c}_{\text {in }}(i, j)<c_{\text {thresh }} \\ 1 & \text { otherwise }\end{cases}
$$

The ratio of the major and minor axes of the largest flagged region in $\mathbf{c}_{\text {out }}$, quantifies the extent of the aperture problem. By using the axes intercept coordinates the peaks' optimal position is found. This is repeated for block scales $M, N=\{64,32,16,8\}$ where the threshold value adapts to the maximum correlation using $c_{\text {thresh }}=0.9 c_{\max }$.

\subsection{Temporal Strain}

Tendons form an integral part of the musculotendinous unit transmitting the tensions generated in muscle to bone. When the tendon is under load, the deformation is expressed as a strain $\varepsilon$, the change in linear dimension per unit length. Although the strain tensor consists of many elements, we are concerned with only translation, where positive or negative nonzero strain (tensile or compressive respectively) is present only if there is a discontinuous displacement in surrounding tissue. Fig. 1 illustrates the strain at an arbitrary tensile point $P$ that shifts to $P^{\prime}$ at coordinates $x$ and $x^{\prime}$ respectively so that $\delta x$ is deformed to $\delta x^{\prime}$. Since the displacement $u=x^{\prime}-x$ at

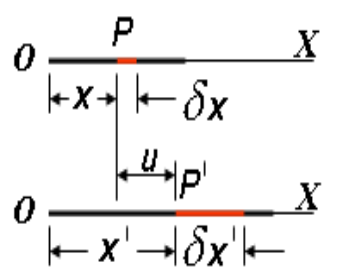
$P \in R$, the local 1D strain is defined as:

$$
\varepsilon=\frac{\delta x^{\prime}-\delta x}{\delta x}=\frac{\delta u}{\delta x}
$$


Using (5) for only frame pairs restricts strain quantification to only single instances as used in other works [1][2]. We use motion trajectories to quantify gradual incremental strain for all blocks in $R$ through any user-required $h$, repeating (5) at each $P$. The result is a temporal history of strain for every position in $f_{t}$ through a sequence. To improve visual understanding we have selected clinical regions of interest (ROI) to illustrate these results, as will shortly be seen in Figs. 2, 5 and 6.

\section{Experimental in vitro Results}

Figs. 2(a) and 2(b) show sample frames of our in vitro groundtruth, a partially cut muscle segment under x-axial linear load. Fig. 2(c) shows a typical trajectory displacement field using a temporal history $h=4$ sampling at $P \times Q=4$ using block scales $M \times N$, where $M, N=\{16,8\}$. Large linear displacements are located at the cut as the muscle is pulled and highlights biased muscle movement to the right as the cut opens, resulting in new trajectories in the region (shown in red) between the cut edges.

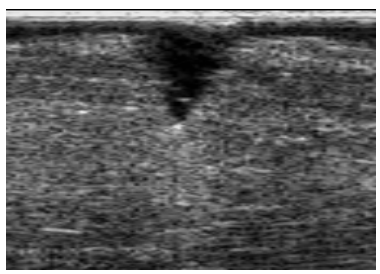

(a) Cut Muscle $f_{6}$ (pre load)

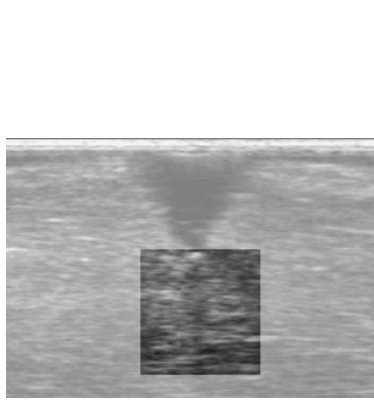

(d) Superimposed ROI

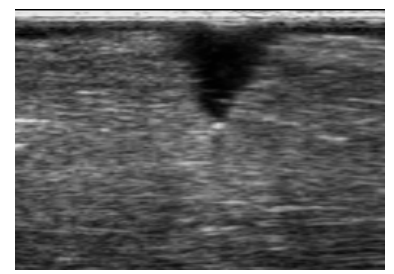

(b) Cut Muscle $f_{10}$ (under load)

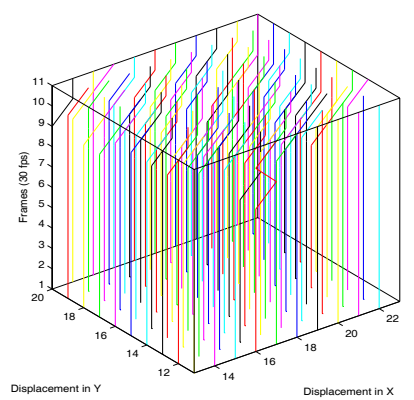

(e) Trajectories for ROI

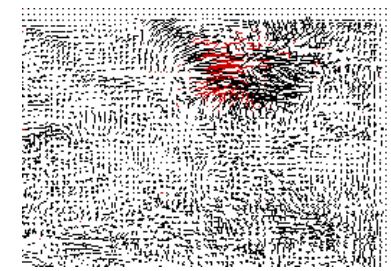

(c) Trajectory Field with $h=4$

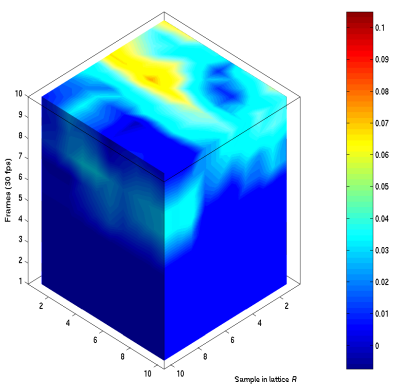

(f) Strain in X showing $h=10$

Figure 2: (a)-(b) In-vitro groundtruth muscle segment, (c) trajectory Field with $h=4$ for $f_{7} \rightarrow f_{11}$, (d) superimposed ROI, (e) 3D Trajectory Field for $f_{1} \rightarrow f_{10}$, (f) strain in X.

Temporal strain calculation was performed on the whole image, while for clarity in Fig. 2(d) we concentrate only on a ROI encapsulating the potential maximum tensile strain concentration (base of the cut) and a sample of relevant frames. With each trajectory marked in a different colour, Fig. 2(e) shows the spatiotemporal trajectory results for frames $f_{1} \rightarrow f_{10}$ for the ROI. Stationary frames $f_{1} \rightarrow f_{6}$ were captured to initialise the sequence, providing a temporal cue to when the load was applied. Results show perfectly linear trajectories in this region. Fig. 2(f) is the final temporal strain result, with zero strain for the initial stationary frames and a peak tensile strain about the cut base. 


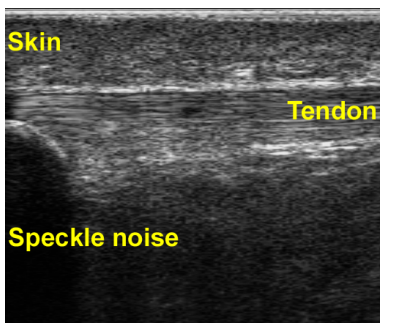

(a) Patella Tendon $f_{10}$

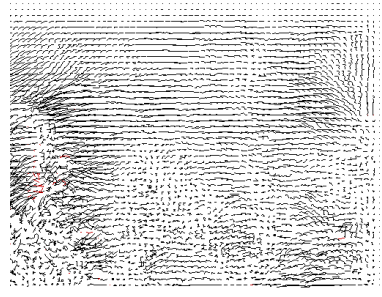

(d) Trajectories $f_{1} \rightarrow f_{10}$

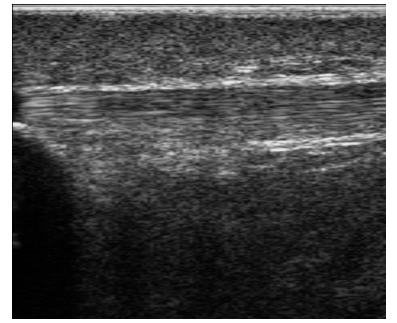

(b) Patella Tendon $f_{20}$

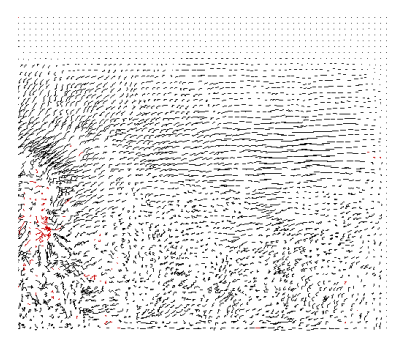

(e) Trajectories $f_{11} \rightarrow f_{20}$

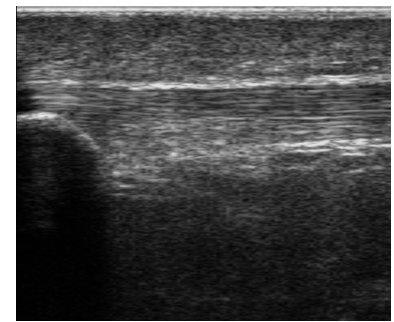

(c) Patella Tendon $f_{30}$

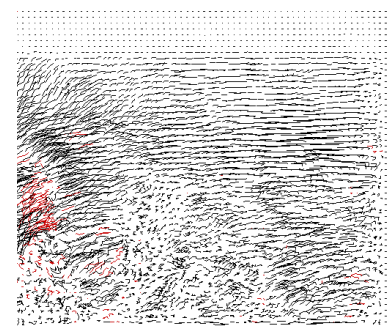

(f) Trajectories $f_{21} \rightarrow f_{30}$

Figure 3: (a)-(c) In vivo patella tendon for $f_{t=10,20,30}$, (d)-(f) displacement trajectory fields, with $h=10$, after probe registration (new object trajectories shown in red).

\section{Experimental in vivo Results}

Figs. 3(a)-3(c) are longitudinal sagittal sections of the patella tendon, traversing left to right from extension to flexion in 1 second and captured at $30 \mathrm{~Hz}$. Trajectory fields in Figs. 3(d)-3(f) were produced by sampling at $P \times Q=8$ using multiple block scales $M \times N$, where $M, N=\{64,32,16,8\}$, with $h=10$. Using the same settings, Figs. 4(a)-4(c) are longitudinal sections of the achilles tendon, with trajectory fields in Figs. 4(d)-4(f).

All trajectory fields are post probe motion registration using (3), resulting in approximate stationarity in the upper skin region so that throughout the sequence this region is constrained to $\mathbf{d} \approx 0$. All trajectories show high temporal correlation for all frames in the sequence. New trajectories entering the frame (shown in red) appear at image boundaries from the horizontal movement, and several appear in the central plane from minor 3D movement. As expected, the trajectories have highly linear x-axial displacements in both the patella and achilles tendons. Beneath the patella tendon (in the lower regions in Figs. 3(a)-3(c)) is well-defined acoustic speckle noise with movement that mimics the tendon motion. Trajectories in this region show some temporal correlation that illustrates similar displacements to the tendon. Beneath the achilles tendon (in the lower regions in Figs. 4(a)-4(c)) there is greater tissue structure that has some independent motion. Trajectories again illustrate regions of strong correlation corresponding to the captured sequence.

In vivo tendon sequences were captured under a full extension to flexion motion, where the maximum strain potentially exists at each limit. For the patella and achilles tendons, frames $f_{1} \rightarrow f_{15}$ capture the tendon with approximate flexion motion, and $f_{16}$ onwards, extension motion. Figs. 5(a) and 5(d) are clinically-relevant selected regions encapsulating samples of the patella bone tendon interface and tendon respectively. Figs. 


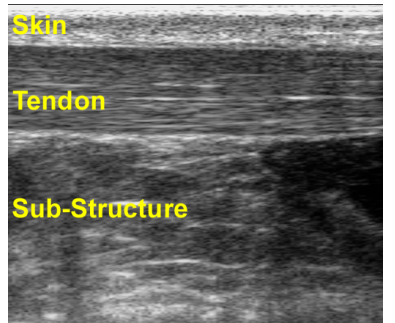

(a) Achilles Tendon $f_{10}$

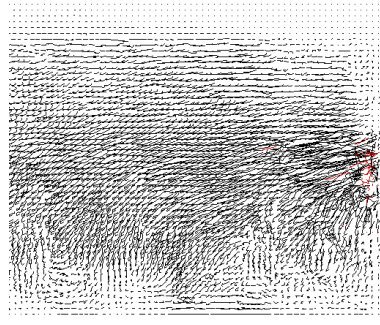

(d) Trajectories $f_{1} \rightarrow f_{10}$

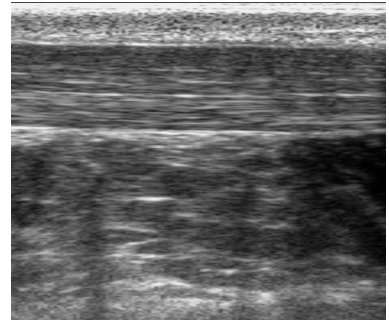

(b) Achilles Tendon $f_{20}$

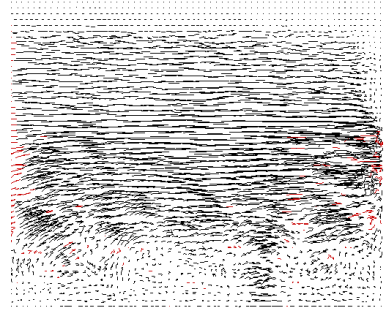

(e) Trajectories $f_{11} \rightarrow f_{20}$

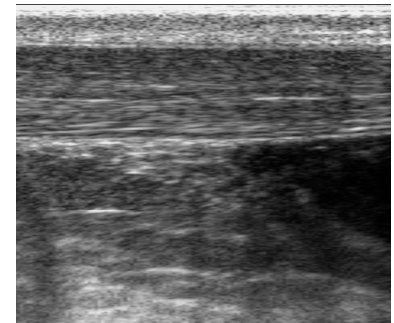

(c) Achilles Tendon $f_{30}$

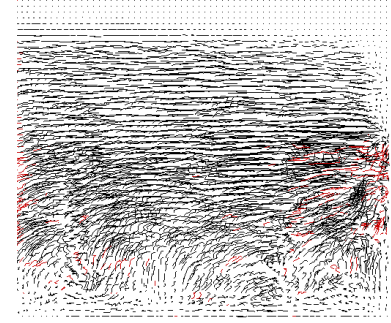

(f) Trajectories $f_{21} \rightarrow f_{30}$

Figure 4: (a)-(c) In vivo achilles tendon for $f_{t=10,20,30}$, (d)-(f) displacement trajectory fields, with $h=10$, after probe registration (new object trajectories shown in red).

5(b) and 5(e) are their associated spatiotemporal trajectories with $h=30$. Figs. 5(c) and 5(f) are the final temporal strain volumes. During extension, the patella bone tendon interface in Fig. 5(a) created large discontinuous trajectories in Fig. 5(b) yielding a relatively high strain concentration $0.5<\varepsilon<1$ and is visible at $f_{20}$ (Fig. 5(c)). However, the ROI throughout the majority of the sequence produced very linear displacements corresponding to negligible strain (Fig. 5(f)). The two regions taken along the longitudinal axis of the tendon also show temporal correlation with each other due to the regions being of the same material having corresponding similar forces imposed on them.

The ROI for the achilles tendon in Fig. 6(a) encapsulates a central region of the tendon. Fig. 6(b) is the associated spatiotemporal trajectories with $h=20$ and Fig. 6(c) is the final temporal strain volume for frames $f_{1} \rightarrow f_{20}$. In this frame range a complete extension to flexion movement was captured. As shown in Fig. 4, the trajectories in this ROI and surroundings are continuous with only negligible vertical displacement. The ROI strain history in Fig. 6(c) illustrates low strain through all 20 frames. Strain ranged from an approximate tensile peak of $\varepsilon=0.1$ about $f_{5}$ and $f_{13}$ and a compressive trough of $\varepsilon=-0.05$ at $f_{9}$ matching the underlying trajectories.

Correlation coefficients measure confidence in trajectory accuracy. Using only a single scale block matching approach we observed large coefficient variance for the displacement fields in our musculoskeletal dataset. From using multiple scales we observed that correlation coefficients increased as block scales refined with improving displacements (Fig. 7). Both in vivo patella (blue diamond line) and achilles (black starred line) tendon sequences from final template candidate block matching were over $95 \%$ correlated. For the in vitro groundtruth (red dotted crossed line) the first 6 frames illustrate 100\% correlation due to these having complete stationarity. 


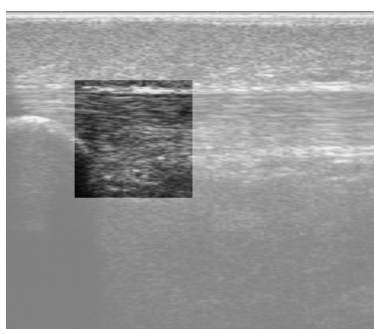

(a) Superimposed ROI $f_{1}$

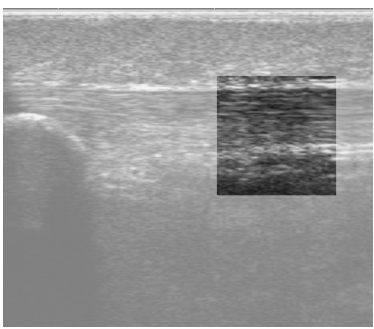

(d) Superimposed ROI $f$

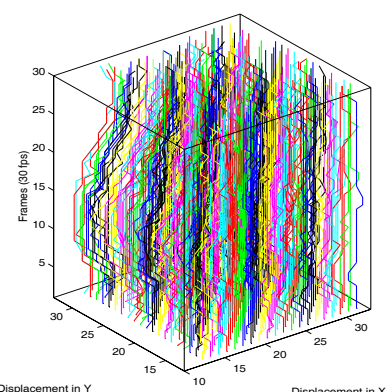

(b) Trajectory Field

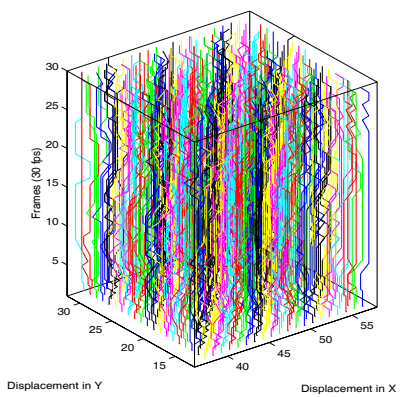

(e) Trajectory Field

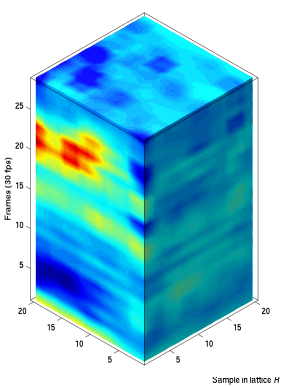

(c) Strain in $X$

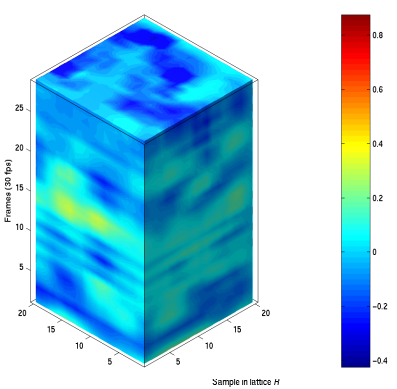

(f) Strain in $\mathrm{X}$

Figure 5: Two ROIs with varying strain history - (a) bone to tendon interface region, (b) trajectory field $(h=30)$, (c) strain in X $(h=30)$, (d) patella tendon region, (e) trajectory field $(h=30)$, (f) strain in X $(h=30)$.

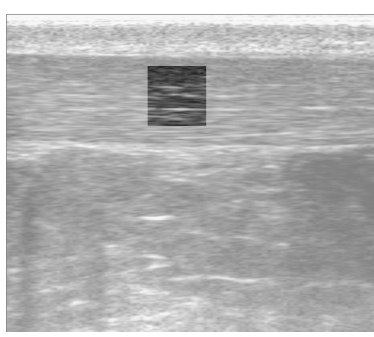

(a) Superimposed ROI $f_{1}$

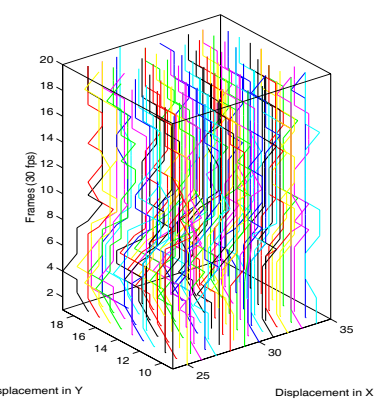

(b) Trajectory Field

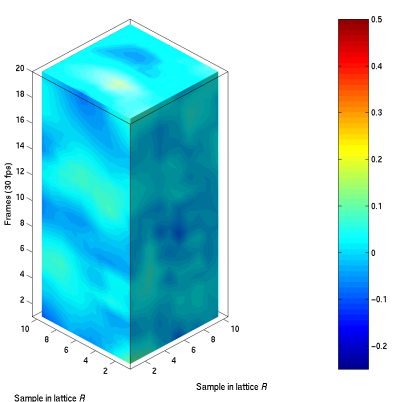

(c) Strain in $\mathrm{X}$

Figure 6: (a) Achilles tendon region, (b) trajectory field $(h=20)$, (c) strain in $\mathrm{X}(h=20)$.

An example of probe motion correction showing significant probe movement was demonstrated for the patella tendon was seen in Fig. 3(f), frames $f_{21} \rightarrow f_{30}$. Figs. 8(a) and 8(b) compare both probe translational displacement results and entering/exiting trajectory updates (normalised), for both patella and achilles tendons. Note, these are shown on the same graphs but are independent. Throughout, the patella tendon sequence 
probe y-axial displacement was approximately zero, with any supported deviations potentially due to probe pressure. After $f_{20}$, x-axial displacement increased to about 3 pixels, with (3) used for trajectory correction, resetting approximate stationarity in the skin region. By determining $\delta_{x y}^{\mu}$ at each frame pair we dynamically update the amount of correction. The achilles tendon was captured with the probe stationary throughout acquisition (Fig. 8(b)). Both sequences illustrate the high temporal trajectory update correlation as new features appear as existing features move out of plane (Fig. 8), from the applied extension flexion motion, with peaks about the limits of the motion.

Probe movement from displacements between skin and probe surface yielded high accuracy once an optimum section was defined. Poor region selection directly affects the registration accuracy, but the mean displacement was sufficient in smoothing spurious values. Consequent registration results were encouraging especially without any prior knowledge from transducer position sensors or definite fixed landmarks.

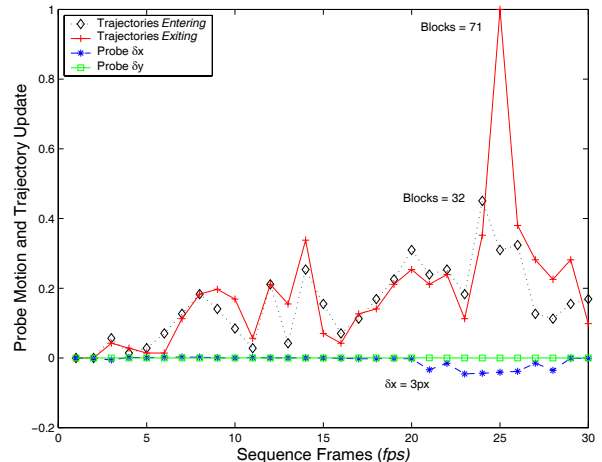

(a) Patella Tendon Statistics

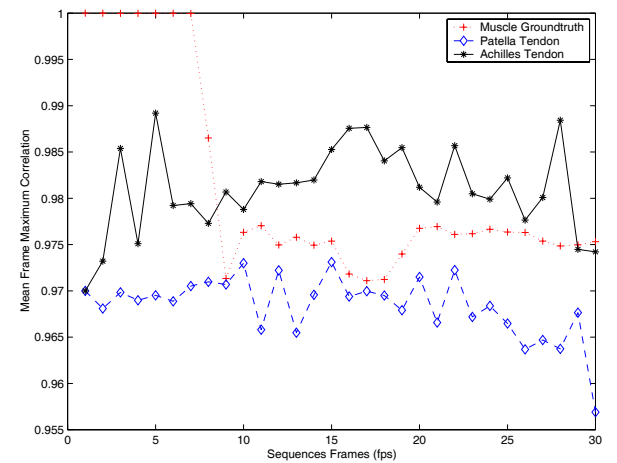

Figure 7: Multiscale NCC Trajectory Confidence $h=1$.

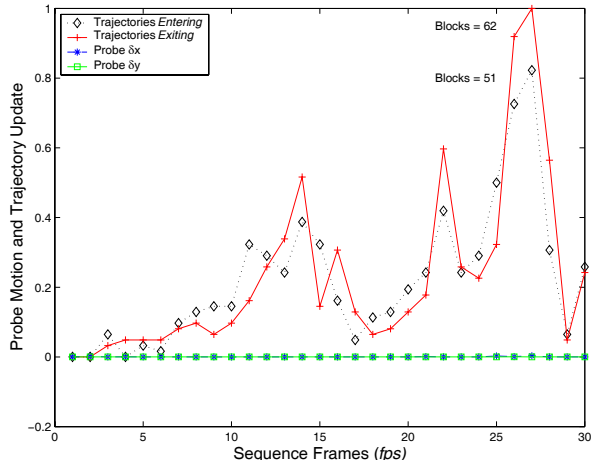

(b) Achilles Tendon Statistics

Figure 8: (a)-(b) Probe displacement and trajectory update.

This work will contribute significantly to soft tissue biomechanic understanding. Currently there is a considerable lack of both experimentally verified material properties for these tissues and experimental validation of analytical and numerical models of the behaviour of organs such as tendons, muscles, intervertebral discs and brains. By quantifying strain, tissue differentiation can be achieved between soft and hard tissue since they react differently to external pressure. The two basic responses to applied pressure are displacement and deformation. Soft tissue tends to deform (undergo nonuniform displacement) while hard tissue displaces uniformly (translates with rigid body motion) in response to the same external forces. With our strain history further work will aim to segment strain to classify hard and soft tissues by exploiting the relative amount of dis- 
placement deformation for the same applied pressure. Since we can now quantify internal motions and deformations within intact structures, it is possible to make direct comparison of model predictions and experimental results at any point within a specimen. Feedback from biomechanic experts has been that this work represents a major step forward in their field, that until now has relied solely on measurements of surface motion and strain.

\section{Conclusion}

We have presented a novel extension to interframe displacements by developing motion trajectories that offer both improved displacement knowledge and yield unique strain history in ultrasound sequences. The trajectory fields proved invariant to a range of capture rates and object movements, whereas interframe displacements only analyse user specific frame pairs. Also regions of tissue can be tracked through typical ultrasound machine aquisition length sequences, eliminating the notion of mere frame matching, and improving temporal coherence. By correcting for probe motion and updating the trajectory field with new objects entering and exiting from the 3D scanned body, we demonstrated a robust 2D speckle tracking technique to understand dynamic musculoskeletal ultrasound by quantifying both trajectories and strain histories.

\section{References}

[1] L Bohs, B Friemel, and G Trahey. Experimental velocity profiles and volumetric flow via 2D speckle tracking. Ultrasound in Med \& Biol, 21(7):885-898, 1995.

[2] D Cooper and J Graham. Estimating motion in noisy, textured images: optical flow in medical ultrasound. In 7th British Machine Vision Conference, pages 585-594, 1996.

[3] J Ellis, J Teh, and P Scott. Ultrasound of tendons. Mini-symposium: Musculoskeletal Ultrasound, 14(2):223-228, 2002.

[4] G Mailloux, F Langois, P Simard, and M Bertrand. Restoration of the velocity field of the heart from 2D echocardiograms. IEEE Trans on Medical Imaging, 8(2):143-153, 1989.

[5] A Morsy and O Ramm. 3D ultrasound tissue motion tracking using correlation search. IEEE Trans on Ultrasonics, Ferroelectrics, and Frequency Control, 20:151-159, 1998.

[6] D Nair and L Wenzel. Image processing and low-discrepency sequences. SPIE Advanced Signal Processing Algorithms, Architectures, and Implementations, 3807(6):102-111, 1999.

[7] J Revell, M Mirmehdi, and D McNally. Variable sized block matching for in vivo musculoskeletal motion analysis. Accepted in Visual Information Engineering,, 2003.

[8] G Treece, R Prager, A Gee, and L Berman. Correction of probe pressure artifacts in freehand 3D ultrasound. In MICCAI, pages 283-290. LNCS 2208, Springer, 2001.

[9] F Valckx and J Thijssen. Characterisation of echographic image texture by cooccurence matrix parameters. Ultrasound in Med \& Biol, 23(4):559-571, 1997.

[10] T Varghese, E Konofagou, J Ophir, S Alam, and M Bilgen. Direct strain estimation in elastography using spectral cross-correlation. Ultrasound in Med \& Biol, 26(9):1525-1537, 2000.

[11] C Wang, J Deng, G Ateshian, and C Hung. An automated approach for direct measurement of 2D strain distributions within articular cartilage under unconfined compression. Journal of Biomechanical Engineering, 124:557-567, 2002.

[12] F Yeung, S Levinson, and K Parker. Multilevel and motion-based ultrasonic speckle tracking algorithms. Ultrasound in Med \& Biol, 24(3):427-441, 1998. 